Main Outcome Measurements An observation form was developed and evaluated containing five categories: (I) general match and player information, (II) localisation of the injury on the pitch, (III) game situation and player/opponent behaviour, (IV) injury mechanism and (V) injured body location.

Results Of the 857 moderate and severe match injuries, 345 $(40.3 \%)$ were clearly identified in the video footage and included to the analysis. Of these, almost half were contact injuries (49.3\%), 23.2\% non-contact injuries and the remaining $27.5 \%$ indirect-contact injuries. Most contact injuries were caused by collisions with the opponent (46.5\%); non-contact injuries were commonly caused by structural overexertion (71.3\%). Finally, nine recurrent comprehensive injury patterns were identified and described.

Conclusions Future preventive approaches should prepare players for the identified recurrent injury situations to reduce injury burden in professional football. One of the main findings was that own tackles are football-specific actions with a high risk for injuries, particularly for knee injuries. Thus, player behaviour and technique skills might present areas with considerable preventive potential. The resulting video database can be used in coaching education to demonstrate recurrent match situations with a high risk for injuries.

\section{PREVALENCE OF HIP AND GROIN PAIN AND CHANGES IN HIP AND GROIN OUTCOME SCORE OVER A SEASON IN ELITE GAELIC ATHLETIC ASSOCIATION PLAYERS}

\footnotetext{
${ }^{1,2}$ Enda King, ${ }^{1,2}$ Chris Richter, ${ }^{5}$ Kristian Thorborg, ${ }^{1,3}$ Andrew Franklyn-Miller, ${ }^{1,4}$ Eanna Falvey, 'James O'Donovan. 'Sports Surgery Clinic, Dublin, Ireland; ' ${ }^{2}$ nniversity of Roehampton, London, UK; ${ }^{3}$ University of Melbourne, Melbourne, Australia; ${ }^{4}$ University College Cork, Cork, Ireland; ${ }^{5}$ Sports Orthopaedic Research Centre Copenhagen, Copenhagen, Denmark
}

\subsection{6/bjsports-2021-IOC.6}

Background Athletic hip and groin pain is common in the Gaelic Athletic Association (GAA) but its prevalence at elite level is unknown. The changes in Hip and Groin Outcome Score (HAGOS) over a season in and the ability of HAGOS to identify those athletes who will go onto develop hip and groin symptoms has not been reported previously.

Objective The aim of this study was to report the prevalence of hip and groin symptoms and the changes in HAGOS score across a season in elite GAA players and examine the relationship between pre-season HAGOS scores and self-reported groin pain during that season.

Design Retrospective cohort study.

Participants There were 1241 elite intercounty GAA players who participated at the start of the 2017 and 2018 seasons.

Interventions (or Assessment of Risk Factors) Online Questionnaire.

Main Outcome Measurements The HAGOS questionnaire and self reported incidence of groin pain in previous season.

Results There was a high prevalence of hip and groin pain reported in elite GAA athletes over the course of a season (36\%). There was no change on average across all the HAGOS subscales apart from Physical Activity which had a medium effect size change $(\mathrm{d}=0.44)$ with 12 to $22 \%$ of athletes showing a decrease greater than minimal detectable change across HAGOS subscales over the course of a season and $15-34 \%$ showing an increase. There was poor ability to predict the incidence of hip and groin pain using pre-season HAGOS (AUC $=0.57$ to 0.67 ).
Conclusions There is a high prevalence of hip and groin pain in elite GAA athletes with group HAGOS masking individual changes across cohort. There is poor ability of pre-season HAGOS to predict incidence of hip and groin pain in elite GAA athletes in the subsequent season.

\section{EPIDEMIOLOGY OF INJURY IN ENGLISH SCHOOLBOY RUGBY UNION}

${ }^{1}$ Matthew Hancock, ${ }^{1}$ Simon Roberts, ${ }^{1}$ Craig Barden, ${ }^{1}$ Carly McKay, ${ }^{2}$ Simon Kemp,

1,2Keith Stokes. 'University of Bath, Bath, UK, ${ }^{2}$ England Rugby, London, UK

10.1136/bjsports-2021-IOC.7

Background There is a perceived high risk of injury within youth rugby, but the available evidence in this population is limited. Understanding injury rates and patterns can inform injury reduction strategies.

Objective To describe the incidence and severity of schoolboy rugby injuries and determine whether there are differences between age groups.

Design A two-season (2017-19) prospective cohort study.

Setting English secondary schools.

Participants Schoolboy teams in the under-13 (U13), under-15 (U15) and under-18 (U18) age groups.

Assessment of Risk Factors Match exposure and the severity (days lost), type and event associated with 24-hour time-loss injuries.

Main Outcome Measures Injury incidence (injuries/1000h) and burden (days lost/1000h).

Results 11,706 player-hours and 379 match injuries from 66 teams were collected. The U18 age group had a significantly higher injury incidence $(37.2$ injuries/1000h, 95\% CI: 33.1 to 41.8) than the U15's (24.7, 95\% CI: 19.8 to 30.8) and U13's (20.8, 95\% CI: 13.6 to 31.9) $(\mathrm{P}<0.01)$, which were not significantly different $(\mathrm{P}=0.24)$. The mean severity was 29 days lost (95\% CI: 26 to 33) for U18, 31 (95\% CI: 25 to 39) for U15 and 20 (95\% CI: 13 to 31) for U13. Injury burden differed significantly between all groups (U18, 1085 days/1000h, 95\% CI: 965 to 1220 ; U15, 767, 95\% CI: 615 to 956; U13, 423, 95\% CI: 276 to 648; $\mathrm{P}<0.01)$. Contact events accounted for $86 \%$ of all injuries, with the tackle accounting for $56 \%$. This was the most common event associated with injury at U18 (22.2 injuries/1000h), U15 (11.4/1000h) and U13 (10.4/ $1000 \mathrm{~h})$. The most common injury type was concussion at U18 (9.0 injuries/1000h) and U15 (5.1/1000h) and bruising/haematoma $(5.2 / 1000 \mathrm{~h})$ at $\mathrm{U} 13$.

Conclusions The U18 age group had the highest injury incidence and burden. The tackle was the most common injury event and should be the focus of further investigation or intervention.

\section{A COMPARISON OF INJURIES BETWEEN MALE AND FEMALE AMATEUR RUGBY UNION PLAYERS}

\footnotetext{
${ }^{1,2}$ Caithriona Yeomans, ${ }^{1,2}$ Thomas M Comyns, ${ }^{2,3}$ Roisin Cahalan, ${ }^{1,2}$ Giles D Warrington, ${ }^{1,2}$ Andrew J Harrison, ${ }^{4}$ Helen Purtill, ${ }^{1}$ Mark Lyons, ${ }^{1,5}$ Mark J Campbell, ${ }^{2,3}$ Liam G Glynn, 1,2lan C Kenny. 'Department of Physical Education and Sport Sciences, University of Limerick, Limerick, Ireland; ${ }^{2}$ Health Research Institute, University of Limerick" Limerick, Ireland; ${ }^{3}$ School of Allied Health, University of Limerick, Limerick, Ireland; ${ }^{4}$ Department of Mathematics and Statistics, University of Limerick, Limerick, Ireland; '5 Lero, The Irish Software Research Centre, University of Limerick, Limerick, Ireland
}

10.1136/bjsports-2021-IOC.8 
Background Rugby participation rates are rising, particularly in the female game where a $60 \%$ increase in player numbers was observed from 2013 to 2017. Despite the recent growth, the female amateur game is lacking comprehensive long-term injury surveillance.

Objective To compare injuries in male and female amateur Rugby Union.

Design Prospective cohort study.

Setting Irish amateur clubs, during seasons 2017/18 ( $\mathrm{n}=15$ male clubs, 4 female) and 2018/19 ( $n=25$ male clubs, 7 female).

Participants Male $(n=958)$ and female $(n=234)$ amateur players.

Independent Variables Match exposure.

Main Outcome Measurements Match injury incidence and severity.

Results Overall incidence rates were 47.7 and 35.4/1,000 player hours for males and females respectively. Similarities existed between males and females regarding common diagnoses and injury occurrence, with 58\% of injuries occurring during the tackle. Concussion and ankle lateral ligament injuries were the most common diagnoses for both males (5.5 and 4.1/1,000 player hours) and females (5.5 and 3.9/1,000 player hours). However differences showed females suffering more injuries in the ruck compared to males (6.1 vs $3.8 / 1,000$ player hours) while males sustained more non-contact injuries compared to females (4.7 vs $1.4 / 1,000$ player hours). Females showed an earlier injury occurrence in the $2^{\text {nd }}$ quarter (9.4/ 1,000 player hours) plateauing into the $3^{\text {rd }}$ and $4^{\text {th }}$ quarters, whereas males had a $3^{\text {rd }}$ quarter injury peak $(15.2 / 1,000$ player hours). Concussion had the highest injury burden in males (190days/1,000 player hours), while anterior cruciate ligament injuries had the highest burden (307days/1,000 player hours) in females.

Conclusions Long-term prospective injury surveillance is vital to inform targeted prevention strategies. The earlier occurrence of injury in females should be investigated further to determine whether player substitution strategies may decrease injuries. Prevention strategies incorporating neuromuscular training should be considered, given the high rate of ankle ligament injuries in both males and females, and the burden of knee ligament injuries in females.

\section{EFFECTS OF A STRENGTH AND PROPRIOCEPTIVE TRAINING PROGRAMME ON NECK FUNCTION AND CONCUSSION INJURY RISK IN ELITE SCOTTISH RUGBY UNION PLAYERS}

${ }^{1}$ Stuart Bailey, ${ }^{1}$ Russell Martindale ${ }^{2}$ Lars Engebretsen, ${ }^{3}$ Jen Sweeting ${ }^{3}$ Jared Deacon ${ }^{3}$ Florence Laing, ${ }^{3}$ Chris Leck, ${ }^{1,4}$ Debbie Palmer. ${ }^{1}$ School of Applied Sciences, Edinburgh Napier University, Edinburgh, UK; ${ }^{2}$ Oslo Sports Trauma Research Center, Oslo, Norway; ${ }^{3}$ Scottish Rugby Union, Edinburgh, UK; ${ }^{4}$ Institute for Sport, PE and Health Sciences, Moray House School of Education and Sport, Edinburgh, UK

\subsection{6/bjsports-2021-IOC.9}

Background Cervical musculature function (strength, endurance, and proprioception) may be a modifiable risk factor for concussion in contact sports. The ability to enhance neck function and reduce concussive risk needs to be determined.

Objective To assess the efficacy of a training programme to enhance neck function and lower match concussion injury risk in rugby union players.
Design Repeated-measures intervention with control.

Setting Premiership rugby union players in Scotland during the 2018/19 season.

Patients (or Participants) Premiership players selected for Scotland Rugby academies (intervention group; INT; $\mathrm{n}=30$ ) or those with their clubs (control group; CON; $n=20$ ).

Interventions (or Assessment of Risk Factors) A neck training programme was implemented twice per week during the 2018/19 season for INT, while CON performed no systematic neck training.

Main Outcome Measurements For both INT and CON neck strength (maximal voluntary contraction; MVC), endurance (exercise capacity; TTF) and proprioception pre- and post-season, and match concussion injury incidence were recorded.

Results Left and right cervical flexion MVC force and flexion TTF all significantly increased in the intervention group $(p<0.001)$, with no significant change in the control group. While there were increases in cervical flexion and extension MVC force in both groups from pre to post-season, there was a significantly greater increase amongst the intervention group $(p<0.05)$. Concussion incidence was lower in INT versus CON (INT: 7.7/1000 match hours; CON: 18.4/1000 match hours). However, this was not a significant alteration in risk (incidence rate ratio: $0.42 ; 95 \%$ CI: $0.08-2.1)$.

Conclusions The neck function programme increased cervical MVC force and flexion exercise capacity, beyond any changes induced by a season of rugby union. The intervention group also had a lower incidence of concussion across the season. This pilot study shows good promise and highlights the need for further investigation.

\section{COMPARISON OF INJURIES AND ILLNESSES BETWEEN REGULAR COMPETITION AND SHORT-TERM MATCH CONGESTION DURING A FULL SEASON IN ELITE MALE PROFESSIONAL BASKETBALL}

\footnotetext{
1,2Steven Doeven, ${ }^{1}$ Michel S Brink, 'Barbara Huijgen, ${ }^{2}$ Johan de Jong, ${ }^{1}$ Koen Lemmink. ${ }^{1}$ Center for Human Movement Sciences, University of Groningen, University Medical Center Groningen, the Netherlands, Groningen, Netherlands; ' 5 Shool of Sport Studies, Hanze University of Applied Sciences, the Netherlands, Groningen, Netherlands
}

\subsection{6/bjsports-2021-IOC.10}

Background It is crucial to balance load and recovery during short-term match congestion in basketball. Currently, it is unknown if higher total load during short-term match congestion lead to higher injury and illness rates.

Objective Aim of this study was to compare injuries and illnesses and total weekly load during 1-match weeks compared to $\geq 2$-match weeks in basketball.

Design During this prospective observational study, players were monitored during a full season.

Setting Two basketball teams participating in the domesticleague championship, CUP matches and Euro league were followed.

Patients (or Participants) Sixteen elite male professional basketball players participated in this study. Characteristics of the players were $($ mean $\pm S D)$ : age $24.8 \pm 2.0$ years, height 195.8 $\pm 7.5 \mathrm{~cm}$, weight $94.8 \pm 14.0 \mathrm{~kg}$, body fat $11.9 \pm 5.0 \%$ and $\mathrm{VO}_{2 \max } 51.9 \pm 5.3 \mathrm{~mL} \cdot \mathrm{kg}^{-1} \cdot \mathrm{min}^{-1}$. 